\title{
OFDM-Based Generalized Optical MIMO
}

\author{
Chen Chen, Member, IEEE, Xin Zhong, Shu Fu, Xin Jian, Min Liu, Helin Yang, Member, IEEE, \\ Arokiaswami Alphones, Senior Member, IEEE, and H. Y. Fu
}

\begin{abstract}
The combination of multiple-input multiple-output (MIMO) transmission and orthogonal frequency division multiplexing (OFDM) modulation has been shown to be an effective way to substantially enhance the capacity of bandlimited optical wireless communication (OWC) systems. In this paper, we propose four OFDM-based generalized optical MIMO techniques for intensity modulation/direct detection (IM/DD) OWC systems, including OFDM-based frequency-domain generalized spatial modulation (FD-GSM), frequency-domain generalized spatial multiplexing (FD-GSMP), time-domain generalized spatial modulation (TD-GSM) and time-domain generalized spatial multiplexing (TD-GSMP). For OFDM-based FD-GSM and FDGSMP, spatial mapping is performed in the frequency domain, while it is carried out in the time domain for OFDM-based TDGSM and TD-GSMP. To efficiently estimate both spatial and constellation symbols in each OFDM-based generalized optical MIMO technique, a corresponding maximum-likelihood (ML) detection algorithm is designed. Extensive simulations are conducted to evaluate and compare the performance of the proposed four OFDM-based generalized optical MIMO techniques in a typical indoor environment. Simulation results demonstrate the superiority of OFDM-based TD-GSM and TD-GSMP for various spectral efficiencies of 4,5 and $6 \mathrm{bits} / \mathrm{s} / \mathrm{Hz}$, when a relatively high secondary direct current (DC) bias is adopted.
\end{abstract}

Index Terms-Optical wireless communication, orthogonal frequency division multiplexing, multiple-input multiple-output.

\section{INTRODUCTION}

$\mathbf{I}$ $\mathrm{N}$ recent years, we have witnessed an explosive growth of smart mobile devices in our daily life, which can provide many exciting services such as high definition video streaming, low-latency gaming, virtual/augmented reality (VR/AR) and Internet-of-things (IoT). In order to meet the demand for these data-greedy services, the requirement of mobile data traffic will face an exponential increase in the near future. It was predicted that the existing radio frequency (RF) spectrum will be saturated soon and the higher frequency part of the electromagnetic spectrum, i.e., the optical spectrum including visible light (VL), infrared (IR) and ultra-violet (UV), has revealed great potential to support the ever-increasing mobile data traffic [1]. Owing to its inherent capability to meet the demand of heavy data traffic, optical wireless communication (OWC) has been triggering tremendous interest in both

This work was supported by the National Natural Science Foundation of China under Grant 61901065.

C. Chen, X. Zhong, S. Fu, X. Jian, and M. Liu are with the School of Microelectronics and Communication Engineering, Chongqing University, Chongqing 400044, China (e-mail: \{c.chen, 201912131098, shufu, jianxin, liumin\}@cqu.edu.cn).

H. Yang and A. Alphones are with the School of Electrical and Electronic Engineering, Nanyang Technological University, Singapore 639798, Singapore (e-mail: hyang013@e.ntu.edu.sg; ealphones@ntu.edu.sg).

$\mathrm{H}$. Fu is with the Tsinghua-Berkeley Shenzhen Institute (TBSI), Tsinghua University, Shenzhen 518055, China (e-mail: hyfu@sz.tsinghua.edu.cn). academia and industry [2]. In typical OWC systems, lightemitting diodes (LEDs) or laser diodes (LDs) are usually used as transmitters and photo-diodes (PDs) are employed as receivers. More specifically, intensity modulation with direct detection (IM/DD) is generally adopted for signal transmission in OWC systems, and hence only real-valued and non-negative signals can be successfully transmitted [3]. Nevertheless, the achievable modulation bandwidth of commercial off-the-shelf (COTS) optical elements is relatively small, especially for white LEDs which usually have a 3-dB bandwidth of about several $\mathrm{MHz}$ [4]. In order to enhance the capacity of OWC systems with a relatively limited modulation bandwidth, the system spectral efficiency should be substantially enhanced.

So far, various techniques have been introduced in bandlimited IM/DD OWC systems for spectral efficiency enhancement, among which multiple-input multiple-output (MIMO) transmission and orthogonal frequency division multiplexing (OFDM) modulation are the two most popular ones. For MIMO transmission, several schemes have been widely considered, including repetition coding (RC), spatial modulation (SM) and spatial multiplexing (SMP) [5], [6]. Among them, $\mathrm{RC}$ is the simplest MIMO scheme, which can obtain transmit diversity. SM can be considered as a digitized MIMO scheme, where only a single transmitter is selected to transmit signal at each time instant, while SMP can achieve high multiplexing gain since different transmitters transmit different signals. For OFDM modulation, high-order quadrature amplitude modulation (QAM) constellations can be applied so as to improve the achievable spectral efficiency [7], [8]. Moreover, the combination of MIMO transmission and OFDM modulation has been further shown as an effective way to substantially enhance the spectral efficiency of bandlimited IM/DD OWC systems. For example, the combination of SM and OFDM was reported in [9]-[12], where the spatial mapping can be performed in the frequency domain, i.e., frequency-domain SM (FD-SM), or the time domain, i.e., time-domain SM (TD-SM). In [13]-[15], the combination of SMP and OFDM was also investigated, where different OFDM signals were transmitted in different SMP channels. Nevertheless, simple MIMO schemes such as SM and SMP cannot fully explore the potential of MIMO transmission for spectral efficiency enhancement of bandlimited OWC systems.

Lately, the concept of generalized spatial modulation (GSM) has been proposed to enhance the performance of conventional SM [16]-[18], which has also been applied in OWC systems [19]-[21]. However, the definition of GSM in the current literature is not accurate. Specifically, the activated transmitters are assumed to transmit the same signal in the GSM defined in [16], [17], [20], [21], and meanwhile different signals can be transmitted by the activated transmitters in the GSM defined in 
[18], [19]. In this work, in order to distinguish the difference of these two categories of GSM schemes, we re-define them as follows: for the one where the activated transmitters transmit the same signal, we define it as "GSM"; for the one where the activated transmitters transmit different signals, we define it as "GSMP". Considering the superiority of GSM and GSMP in comparison to conventional MIMO techniques such as SM and SMP, it is of great significance to combine them with OFDM for bandlimited IM/DD OWC systems.

In this paper, we for the first time propose four OFDMbased generalized optical MIMO techniques for bandlimited IM/DD OWC systems, including OFDM-based FD-GSM, FDGSMP, TD-GSM and TD-GSMP. Specifically, spatial mapping in OFDM-based FD-GSM and FD-GSMP is performed in the frequency domain, while it is executed in the time domain for OFDM-based TD-GSM and TD-GSMP. Maximum-likelihood (ML) detection algorithms are designed to efficiently estimate both spatial and constellation symbols for the proposed four OFDM-based generalized optical MIMO techniques. Moreover, for OFDM-based FD-GSM and TD-GSM, maximal-ratio combining (MRC) is followed to achieve maximum transmit diversity. Detailed simulation results are presented to evaluate and compare the performance of the proposed four OFDMbased generalized optical MIMO techniques in a typical indoor environment for different spectral efficiencies.

The rest of this paper is organized as follows. In Section II, we first describe the mathematical model of a general IM/DDbased MIMO-OWC system. In Section III, we propose four OFDM-based generalized optical MIMO techniques for OWC systems. Detailed simulation setup and results are presented in Section IV. Finally, Section V concludes the paper.

Notation: $(\cdot)^{T},(\cdot)^{*}$ and $(\cdot)^{\dagger}$ represent the transpose, conjugated transpose and pseudo inverse of a vector or matrix, respectively. $(\cdot)^{-1}$ represents the inverse of a matrix. $\lfloor\cdot\rfloor$ and $\|\cdot\|_{2}$ denote the floor and modulus operators, respectively.

\section{SySTEM MODEL}

In this section, we describe the mathematical model of a general IM/DD-based MIMO-OWC system equipped with $N_{t}$ LEDs and $N_{r}$ PDs. Let $\mathbf{x}=\left[x_{1}, x_{2}, \cdots, x_{N_{t}}\right]^{T}$ be the transmitted signal vector, $\mathbf{H}$ represent the $N_{r} \times N_{t}$ MIMO channel matrix and $\mathbf{n}=\left[n_{1}, n_{2}, \cdots, n_{N_{r}}\right]^{T}$ denote the additive noise vector. The received signal vector $\mathbf{y}=\left[y_{1}, y_{2}, \cdots, y_{N_{r}}\right]^{T}$ can be obtained by

$$
\mathbf{y}=\mathbf{H x}+\mathbf{n}
$$

where the channel matrix of the $N_{r} \times N_{t}$ MIMO-OWC system can be expressed as follows:

$$
\mathbf{H}=\left[\begin{array}{ccc}
h_{11} & \cdots & h_{1 N_{t}} \\
\vdots & \ddots & \vdots \\
h_{N_{r} 1} & \cdots & h_{N_{r} N_{t}}
\end{array}\right]
$$

In (2), $h_{r t}\left(r=1,2, \cdots, N_{r} ; t=1,2, \cdots, N_{t}\right)$ denotes the direct current (DC) channel gain between the $r$-th PD and the $t$-th LED. Here, we assume that each LED follows the Lambertian radiation pattern and only the line-of-sight (LOS) transmission is considered [22]. Hence, $h_{r t}$ can be calculated as follows:

$$
h_{r t}=\frac{(m+1) \rho A}{2 \pi d_{r t}^{2}} \cos ^{m}\left(\varphi_{r t}\right) T_{s}\left(\theta_{r t}\right) g\left(\theta_{r t}\right) \cos \left(\theta_{r t}\right),
$$

where $m=-\ln 2 / \ln (\cos (\Psi))$ denotes the Lambertian emission order and $\Psi$ is the semi-angle at half power of the LED; $\rho$ and $A$ are the responsivity and the active area of the PD, respectively; $d_{r t}$ is the distance between the $r$-th PD and the $t$-th LED; $\varphi_{r t}$ and $\theta_{r t}$ are the emission angle and the incident angle, respectively; $T_{s}\left(\theta_{r t}\right)$ is the gain of optical filter; $g\left(\theta_{r t}\right)=\frac{n^{2}}{\sin ^{2} \Phi}$ is the gain of optical lens, where $n$ and $\Phi$ are the refractive index and the half-angle field-of-view (FOV) of the optical lens, respectively.

To recover the transmitted signal vector $\mathrm{x}$, zero forcing $(\mathrm{ZF})$ equalization is adopted at the receiver side for MIMO demultiplexing [23], [24]. After applying ZF equalization, the estimate of $\mathbf{x}$ can be obtained as follows:

$$
\hat{\mathbf{x}}=\mathbf{H}^{\dagger} \mathbf{y}=\mathbf{x}+\mathbf{H}^{\dagger} \mathbf{n}
$$

where $\mathbf{H}^{\dagger}=\left(\mathbf{H}^{*} \mathbf{H}\right)^{-1} \mathbf{H}^{*}$ denotes the pseudo inverse of $\mathbf{H}$.

The additive noise in the IM/DD-based MIMO-OWC system usually consists of both shot and thermal noises, which can be reasonably modeled as a real-valued zero-mean additive white Gaussian noise (AWGN) with power $P_{n}=N_{0} B$, where $N_{0}$ and $B$ denote the noise power spectral density (PSD) and the modulation bandwidth, respectively.

\section{OFDM-BASED GENERALIZED OPTICAL MIMO}

In this section, we introduce four OFDM-based generalized optical MIMO techniques, including OFDM-based FD-GSM, FD-GSMP, TD-GSM and TD-GSMP.

\section{A. OFDM-Based FD-GSM}

The first OFDM-based generalized optical MIMO technique is OFDM-based FD-GSM, where the GSM mapping is performed in the frequency domain, i.e., the subcarrier level. Fig. 1(a) illustrates the schematic diagram of the $N_{r} \times N_{t}$ OFDMbased FD-GSM system. For each subcarrier slot, the input bits are first separated and then mapped into a constellation symbol $c$ and a spatial index vector $\mathbf{v}_{F}=\left[v_{1, F}, v_{2, F}, \cdots, v_{N, F}\right]^{T}$, where $v_{i, F} \in\left\{1,2, \cdots, N_{t}\right\}$ and $v_{i, F} \neq v_{j, F}$ if $i \neq j$ for $i, j \in\{1,2, \cdots, N\}$. Without loss of generality, we assume that the spatial indices in $\mathbf{v}_{F}$ are sorted in the ascending order, i.e., $v_{1, F}<v_{2, F}<\cdots<v_{N, F}$. After that, FD-GSM mapping is performed in the subcarrier level, where $N$ out of totally $N_{t}$ OFDM modulators are selected to transmit the same constellation symbol $c$ while the inputs of the other OFDM modulators are set to zeros. Hence, letting $\mathbf{x}_{F}=\left[x_{1, F}, x_{2, F}, \cdots, x_{N_{t}, F}\right]^{T}$ denote the resultant transmit vector of $N_{t}$ OFDM modulators for a given subcarrier slot, $x_{k, F}\left(k=1,2, \cdots, N_{t}\right)$ can be obtained as follows:

$$
x_{k, F}=\left\{\begin{array}{ll}
c, & \text { if } k=v_{i, F} \text { for } i \in\{1,2, \cdots, N\} \\
0, & \text { otherwise }
\end{array} .\right.
$$




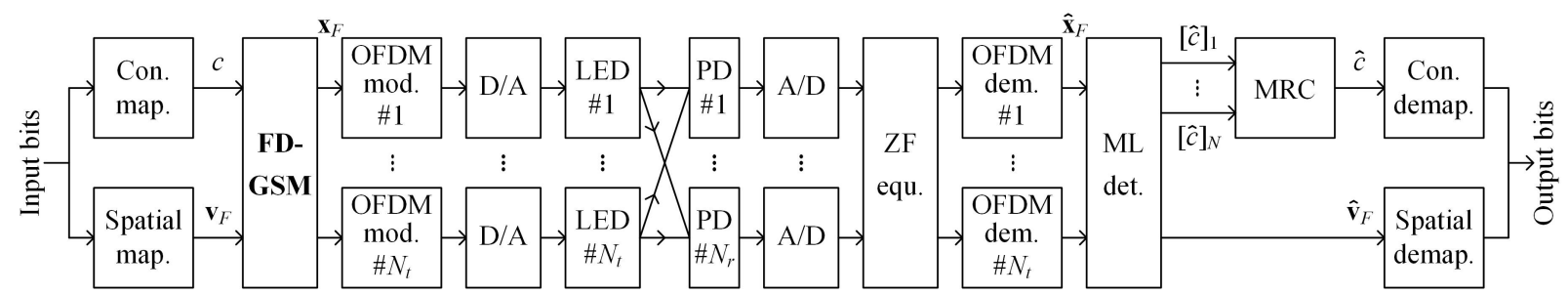

(a) OFDM-based FD-GSM

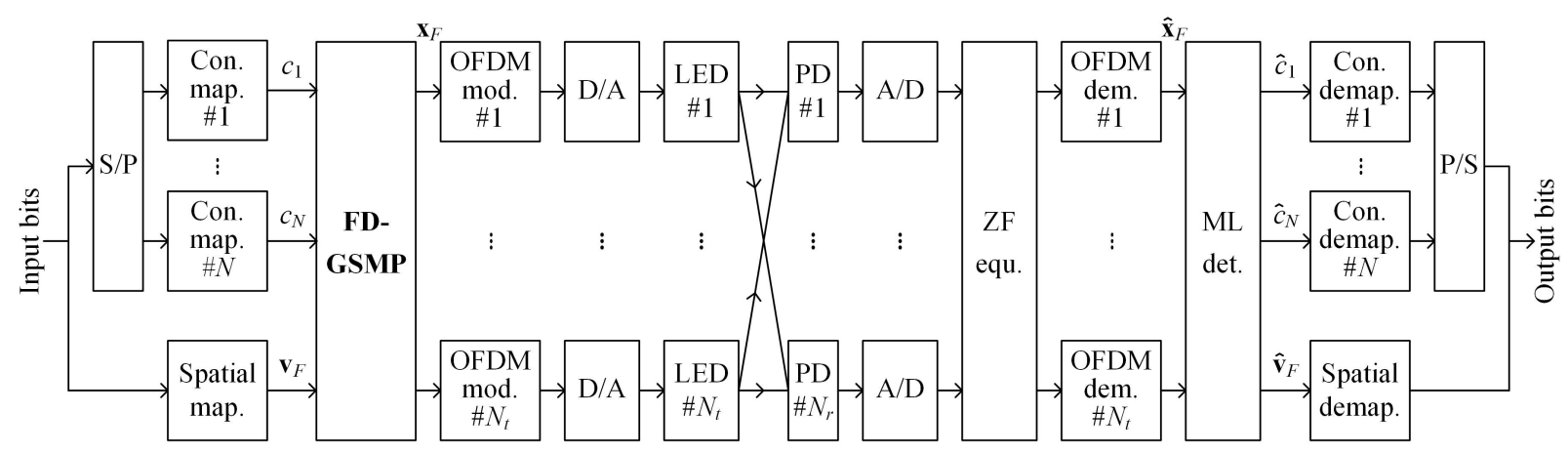

(b) OFDM-based FD-GSMP

Fig. 1. Schematic diagrams of OFDM-based (a) FD-GSM and (b) FD-GSMP. Con.: constellation; map.: mapping; mod.: modulation; equ.: equalization; dem.: demodulation; det.: detection; demap.: demapping.

TABLE I

FD-GSM MAPPING TABLE FOR $N_{t}=4$ AND $N=2$

\begin{tabular}{ccccc}
\hline Spatial bits & OFDM \#1 & OFDM \#2 & OFDM \#3 & OFDM \#4 \\
\hline 00 & $c$ & $c$ & 0 & 0 \\
01 & $c$ & 0 & $c$ & 0 \\
11 & 0 & $c$ & 0 & $c$ \\
10 & 0 & 0 & $c$ & $c$ \\
\hline
\end{tabular}

Table I shows the mapping table of FD-GSM for $N_{t}=4$ and $N=2$. As we can see, taking the spatial bits " 01 " as an example, the corresponding spatial index vector is given by $\mathbf{v}_{F}=[1,3]^{T}$ and hence the OFDM modulators \#1 and \#3 are selected to transmit the same constellation symbol $c$ while the inputs of the OFDM modulators \#2 and \#4 are set to zeros. As a result, the obtained transmit vector can be expressed by $\mathbf{x}_{F}=[c, 0, c, 0]^{T}$. After FD-GSM mapping, the $N_{t}$ parallel data streams are modulated into digital OFDM signals, which are further converted to analog OFDM signals via digital-toanalog (D/A) conversion and subsequently utilized to drive $N_{t}$ LED transmitters. It should be noted that OFDM-based FDGSM with $N=1$ becomes the conventional OFDM-based FD-SM as reported in [11].

Due to the IM/DD nature of typical MIMO-OWC systems, the Hermitian symmetry constraint is usually imposed at the input of inverse fast Fourier transform (IFFT) during OFDM modulation, in order to achieve IM/DD-compatible real-valued OFDM signals [7], [25], [26]. For the OFDM modulation with $L$-point IFFT, the input vector of IFFT can be expressed by

$$
\mathbf{S}=\left[0, S_{1}, \cdots, S_{L / 2-1}, 0, S_{L / 2-1}^{*}, \cdots, S_{1}^{*}\right]^{T} .
$$

It can be found that only $\frac{L}{2}-1$ subcarriers out of totally $L$ subcarriers can be used for valid data transmission because of the imposed Hermitian symmetry constraint. After performing IFFT, a real-valued bipolar time-domain signal can be generated as follows:

$$
s_{l}=\frac{1}{\sqrt{L}} \sum_{k=0}^{L-1} S_{k} \exp \left(\frac{j 2 \pi k l}{L}\right),
$$

where $l \in\{0,1, \cdots, L-1\}$. When $L$ is relatively large (e.g., $L>64$ ), the time-domain signal follows a zero-mean Gaussian distribution with variance $\sigma^{2}$, i.e., $s_{l} \sim \mathcal{N}\left(0, \sigma^{2}\right)$. To convert the obtained bipolar OFDM signal into a unipolar one for intensity modulation in LED transmitters, a proper DC bias is generally introduced [27]. However, since OFDM signals exhibit relatively high peak-to-average power ratios (PAPRs), zero clipping is usually first executed to remove the excessive large negative samples in order to avoid the use of large DC biases. Hence, the biased and clipped time-domain sample can be given by

$$
s_{l}^{\prime}=\left\{\begin{array}{ll}
0, & s_{l}<-b \\
s_{l}+b, & s_{l} \geq-b
\end{array},\right.
$$

where $b=\alpha \sigma$ denotes the added DC bias with $\alpha$ being the proportionality constant. The DC bias in decibels is defined as $b_{\mathrm{dB}}=10 \log _{10}\left(\alpha^{2}+1\right)$.

According to the spatial index vector $\mathbf{v}_{F}$, OFDM modulator selection is carried out in the subcarrier level. Since only $\frac{L}{2}-1$ subcarriers can be used for valid data transmission for a $L$ point IFFT in OFDM modulation, OFDM modulator selection can only be performed with respect to these $\frac{L}{2}-1$ subcarriers. As a result, the spectral efficiency (bits/s/Hz) of OFDM-based 


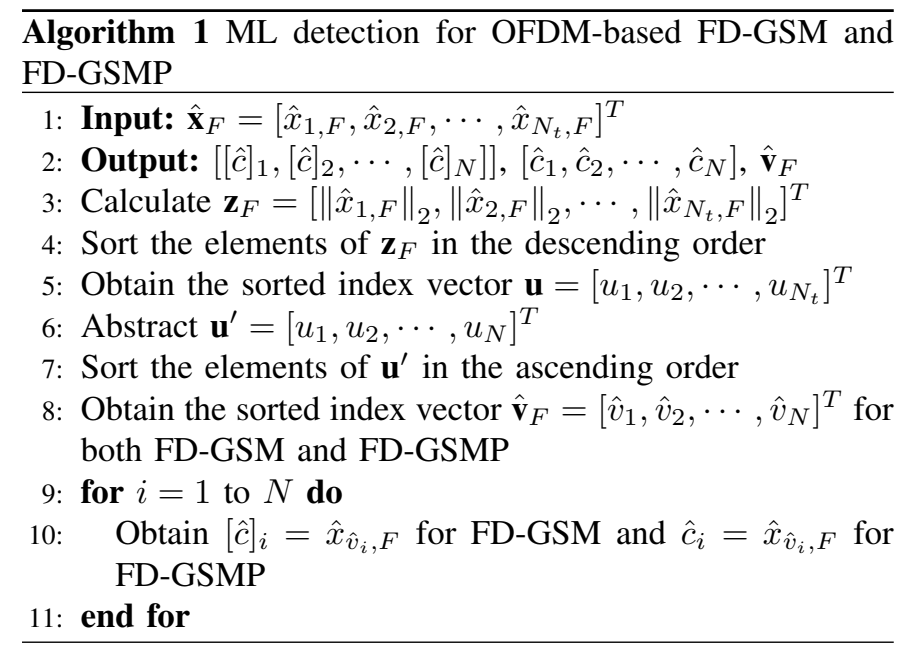

FD-GSM with $N_{t}$ LEDs and $M$-ary constellation is given by

$$
\begin{aligned}
\eta_{\text {FD-GSM }} & =\frac{\frac{L}{2}-1}{L}\left(\log _{2}(M)+\left\lfloor\log _{2}\left(C\left(N_{t}, N\right)\right)\right\rfloor\right) \\
& \approx \underbrace{\frac{1}{2} \log _{2}(M)}_{\text {constellation }}+\underbrace{\frac{1}{2}\left\lfloor\log _{2}\left(C\left(N_{t}, N\right)\right)\right\rfloor}_{\text {spatial }},
\end{aligned}
$$

where $\lfloor\cdot\rfloor$ denotes the floor operator which outputs an integer smaller or equal to its input value and $C(\cdot, \cdot)$ represents the binomial coefficient. The first term in (9) is contributed by the constellation symbols carried by $\frac{L}{2}-1$ subcarriers, while the second term is contributed by the spatial symbols. Moreover, the scaling factor $\frac{1}{2}$ corresponding to both the constellation and spatial symbols is due to the imposed Hermitian symmetry constraint in OFDM modulation [28].

At the receiver side, the radiated light is detected by $N_{r}$ PDs and the resultant outputs are digitized via analog-to-digital (A/D) conversion. After ZF equalization and parallel OFDM demodulation, the estimate of the transmit vector $\mathbf{x}_{F}$ can be obtained by $\hat{\mathbf{x}}_{F}$, and ML detection is further performed to estimate the transmitted constellation symbol vector and spatial index vector. The detailed procedures to perform ML detection for OFDM-based FD-GSM are presented in Algorithm 1. The modulus vector $\mathbf{z}_{F}=\left[\left\|\hat{x}_{1, F}\right\|_{2},\left\|\hat{x}_{2, F}\right\|_{2}, \cdots,\left\|\hat{x}_{N_{t}, F}\right\|_{2}\right]^{T}$ is first calculated, where $\|\cdot\|_{2}$ denotes the modulus operator, and then the elements of $\mathbf{z}_{F}$ are sorted in the descending order so as to obtain the sorted index vector $\mathbf{u}=\left[u_{1}, u_{2}, \cdots, u_{N_{t}}\right]^{T}$. Subsequently, the first $N$ elements of $\mathbf{u}$ are abstracted and the corresponding index vector $\mathbf{u}^{\prime}=\left[u_{1}, u_{2}, \cdots, u_{N}\right]^{T}$ can be obtained. We further sort the elements of $\mathbf{u}^{\prime}$ in the ascending order and the sorted index vector yields the estimate of the transmitted spatial index vector, i.e., $\hat{\mathbf{v}}_{F}=\left[\hat{v}_{1}, \hat{v}_{2}, \cdots, \hat{v}_{N}\right]^{T}$. Moreover, the estimate of the transmitted constellation symbol vector, i.e., $\left.\left[[\hat{c}]_{1},[\hat{c}]_{2}, \cdots,[\hat{c}]_{N}\right]\right]$, can be directly obtained by using $\hat{\mathbf{x}}_{F}$ and $\hat{\mathbf{v}}_{F}$. In order to achieve the final estimate of the transmitted constellation symbol $c$, i.e., $\hat{c}$, MRC is adopted to combine the $N$ elements in $\left.\left[[\hat{c}]_{1},[\hat{c}]_{2}, \cdots,[\hat{c}]_{N}\right]\right][5],[29]$. Finally, the output bits can be generated through constellation demapping and spatial demapping.
TABLE II

FD-GSMP MAPPING TABLE FOR $N_{t}=4$ AND $N=2$

\begin{tabular}{ccccc}
\hline Spatial bits & OFDM \#1 & OFDM \#2 & OFDM \#3 & OFDM \#4 \\
\hline 00 & $c_{1}$ & $c_{2}$ & 0 & 0 \\
01 & $c_{1}$ & 0 & $c_{2}$ & 0 \\
11 & 0 & $c_{1}$ & 0 & $c_{2}$ \\
10 & 0 & 0 & $c_{1}$ & $c_{2}$ \\
\hline
\end{tabular}

\section{B. OFDM-Based FD-GSMP}

In OFDM-based FD-GSM, multiple OFDM modulators can be selected to transmit the same constellation symbol and thus transmit diversity can be achieved in the frequency domain. Nevertheless, the achievable spectral efficiency contributed by constellation symbols is still the same as that of conventional SM, which limits the overall achievable spectral efficiency. In order to achieve enhanced overall spectral efficiency, the second OFDM-based generalized optical MIMO technique, i.e., OFDM-based FD-GSMP, is further proposed.

The schematic diagram of the $N_{r} \times N_{t}$ OFDM-based FDGSMP system is shown in Fig. 1(b). As we can see, the input bits are first divided into two streams: one is for constellation mapping and the other is for spatial mapping. The serial bit stream for constellation mapping is further converted into $N$ parallel bit streams via serial-to-parallel ( $\mathrm{S} / \mathrm{P}$ ) conversion, which are then mapped into $N$ parallel constellation symbols. Differing from OFDM-based FD-GSM where the $N$ selected OFDM modulators are used to transmit the same constellation symbol, they are used to simultaneously transmit different constellation symbols in OFDM-based FD-GSMP. Letting $\mathbf{c}=\left[c_{1}, c_{2}, \cdots, c_{N}\right]^{T}$ denote the transmitted constellation symbol vector, for a given subcarrier slot, we can obtain the resultant transmit vector $\mathbf{x}_{F}=\left[x_{1, F}, x_{2, F}, \cdots, x_{N_{t}, F}\right]^{T}$ after FD-GSMP mapping as follows:

$$
x_{k, F}= \begin{cases}c_{i}, & \text { if } k=v_{i, F} \text { for } i \in\{1,2, \cdots, N\} \\ 0, & \text { otherwise }\end{cases}
$$

for $k=1,2, \cdots, N_{t}$. The mapping table of FD-GSMP for $N_{t}=4$ and $N=2$ is given in Table II. Similarly, taking the spatial bits " 01 " as an example, the corresponding spatial index vector is still $\mathbf{v}_{F}=[1,3]^{T}$, but the OFDM modulators $\# 1$ and \#3 are selected to transit constellation symbols $c_{1}$ and $c_{2}$, respectively. Hence, the transmit vector is obtained by $\mathbf{x}_{F}=\left[c_{1}, 0, c_{2}, 0\right]^{T}$. Similarly, OFDM-based FD-GSMP also becomes OFDM-based FD-SM when $N=1$ [11].

For FD-GSMP mapping, $N$ out of $N_{t}$ OFDM modulators are selected to transmit different constellation symbols and hence the spectral efficiency contributed by spatial symbols is the same for FD-GSM and FD-GSMP. However, since $N$ different constellation symbols can be simultaneously transmitted for each subcarrier slot in FD-GSMP, the spectral efficiency contributed by constellation symbols for FD-GSMP becomes $N$ times of that for FD-GSM. Therefore, the spectral efficiency (bits/s/Hz) of OFDM-based FD-GSMP with $N_{t}$ LEDs and $M$ - 


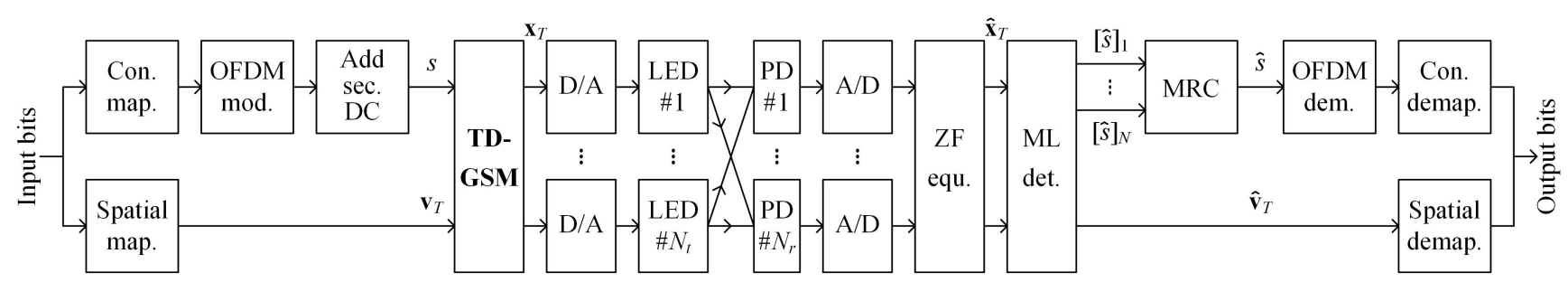

(a) OFDM-based TD-GSM

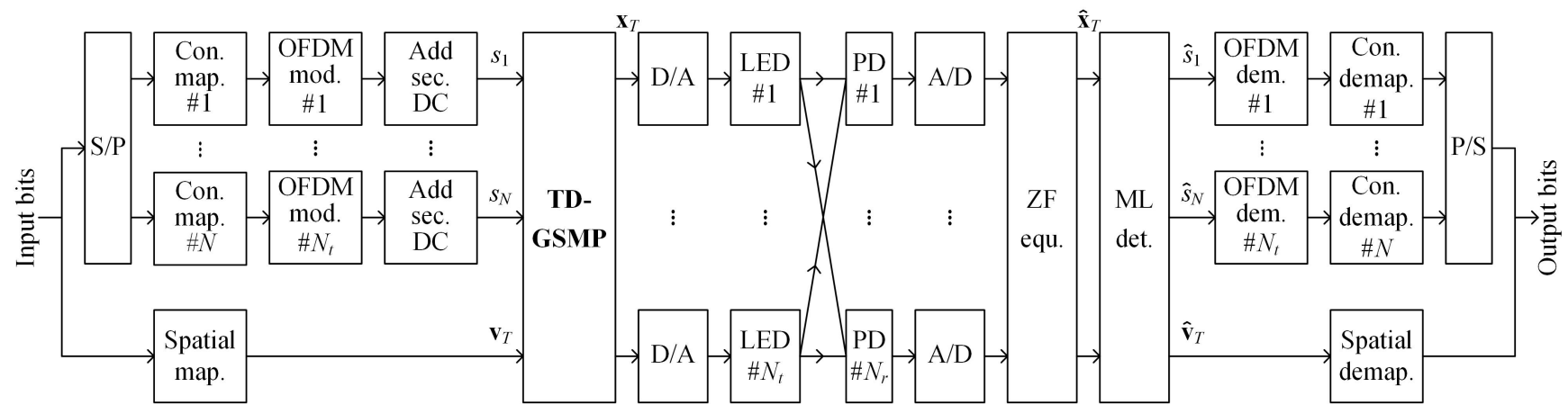

(b) OFDM-based TD-GSMP

Fig. 2. Schematic diagrams of OFDM-based (a) TD-GSM and (b) TD-GSMP. Con.: constellation; map.: mapping; mod.: modulation; sec.: secondary; equ.: equalization; dem.: demodulation; det.: detection; demap.: demapping.

ary constellation is obtained by

$$
\begin{aligned}
\eta_{\text {FD-GSMP }} & =\frac{\frac{L}{2}-1}{L}\left(N \log _{2}(M)+\left\lfloor\log _{2}\left(C\left(N_{t}, N\right)\right)\right\rfloor\right) \\
& \approx \underbrace{\frac{N}{2} \log _{2}(M)}_{\text {constellation }}+\underbrace{\frac{1}{2}\left\lfloor\log _{2}\left(C\left(N_{t}, N\right)\right)\right\rfloor}_{\text {spatial }} .
\end{aligned}
$$

After ZF equalization and parallel OFDM demodulation at the receiver side, ML detection is carried out to estimate the transmitted constellation symbol vector and spatial index vector for OFDM-based FD-GSMP. The detailed procedures of ML detection for OFDM-based FD-GSMP are the same as that of OFDM-based FD-GSM, which can be found in Algorithm 1. The $N$ constellation symbols in the estimated constellation symbol vector $\left[\hat{c}_{1}, \hat{c}_{2}, \cdots, \hat{c}_{N}\right]$ are simultaneously demapped and the obtained parallel constellation bit streams are subsequently converted into a serial bit stream via parallel-to-serial (P/S) conversion. Moreover, the spatial bits can be directly obtained by demapping the estimated spatial index vector $\hat{\mathbf{v}}_{F}$.

\section{OFDM-Based TD-GSM}

In both OFDM-based FD-GSM and FD-GSMP, the spatial information is carried by OFDM modulator selection in the frequency domain. Due to the imposed Hermitian symmetry constraint, only approximately half of all the subcarriers can be utilized to carry valid data and hence OFDM modulator selection can only be performed with respect to these subcarriers. As a result, the spectral efficiency contributed by spatial symbols in both OFDM-based FD-GSM and FD-GSMP is inevitably halved. To eliminate the impact of the imposed Hermitian symmetry constraint and hence achieve enhanced spectral efficiency contributed by spatial symbols, we propose the third OFDM-based generalized optical MIMO technique, i.e., OFDM-based TD-GSM.

Fig. 2(a) shows the schematic diagram of the $N_{r} \times N_{t}$ OFDM-based TD-GSM system, where the spatial information is encoded in the time domain via LED transmitter selection. More specifically, for each time slot, $N$ out of totally $N_{t}$ LED transmitters are selected to transmit the same time-domain sample while the other LEDs are not activated. To successfully encode the spatial information, the transmitted time-domain sample should be non-zero. It can be seen from (8) that the biased and clipped time-domain OFDM signal might have a few zero elements due to zero clipping. When the time-domain samples are zero or very small, the spatial information might be lost. Hence, a secondary DC bias can be added after OFDM modulation so as to guarantee that all the time-domain samples have positive values [11]. After adding a secondary DC bias, (8) can be modified as follows:

$$
s_{l}^{\prime}=\left\{\begin{array}{ll}
\epsilon, & x_{l}<-b \\
x_{l}+b+\epsilon, & x_{l} \geq-b
\end{array},\right.
$$

where $\epsilon=\rho b$ is the value of the secondary DC bias with $\rho$ denoting the ratio between the secondary DC bias (i.e., $\epsilon$ ) and the primary DC bias (i.e., $b$ ).

Letting $s$ and $\mathbf{v}_{T}=\left[v_{1, T}, v_{2, T}, \cdots, v_{N, T}\right]^{T}$ respectively denote the time-domain sample and the spatial index vector, for a given time slot, the resultant transmit vector of $N_{t}$ LED transmitters $\mathbf{x}_{T}=\left[x_{1, T}, x_{2, T}, \cdots, x_{N_{t}, T}\right]^{T}$ after TD-GSM mapping can be obtained by

$$
x_{k, T}=\left\{\begin{array}{ll}
s, & \text { if } k=v_{i, T} \text { for } i \in\{1,2, \cdots, N\} \\
0, & \text { otherwise }
\end{array},\right.
$$


TABLE III

TD-GSM MAPPING TABLE FOR $N_{t}=4$ AND $N=2$

\begin{tabular}{ccccc}
\hline Spatial bits & LED \#1 & LED \#2 & LED \#3 & LED \#4 \\
\hline 00 & $s$ & $s$ & 0 & 0 \\
01 & $s$ & 0 & $s$ & 0 \\
11 & 0 & $s$ & 0 & $s$ \\
10 & 0 & 0 & $s$ & $s$ \\
\hline
\end{tabular}

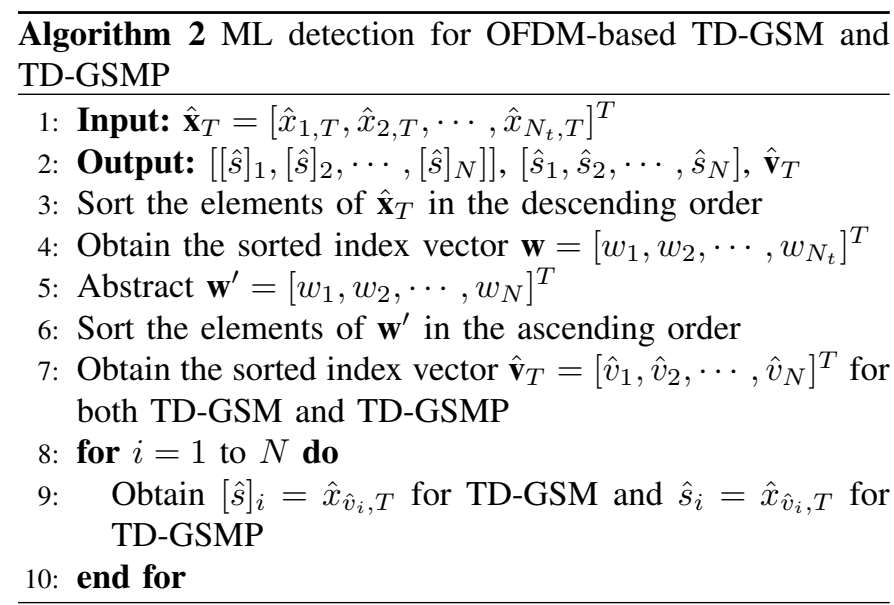

for $k=1,2, \cdots, N_{t}$. The corresponding mapping table of TDGSM for $N_{t}=4$ and $N=2$ can be found in Table III. Taking the spatial bits " 01 " as an example, the spatial index vector is given by $\mathbf{v}_{T}=[1,3]^{T}$ and hence the LED transmitters \#1 and \#3 are selected to transit the same time-domain sample $s$ while the inputs of the LED transmitters \#2 and \#4 are set to zeros. Hence, the obtained transmit vector can be given by $\mathbf{x}_{T}=[s, 0, s, 0]^{T}$. Note that OFDM-based TD-GSM becomes OFDM-based TD-SM when $N=1$ [11].

In OFDM-based TD-GSM, LED transmitter selection can be performed with respect to all the time-domain samples and hence the spectral efficiency contributed by spatial symbols is doubled in comparison to that of both OFDM-based FD-GSM and FD-GSMP. Therefore, the spectral efficiency (bits/s/Hz) of OFDM-based TD-GSM with $N_{t}$ LEDs and $M$-ary constellation can be given by

$$
\begin{aligned}
\eta_{\text {TD-GSM }} & =\frac{\frac{L}{2}-1}{L} \log _{2}(M)+\left\lfloor\log _{2}\left(C\left(N_{t}, N\right)\right)\right\rfloor \\
& \approx \underbrace{\frac{1}{2} \log _{2}(M)}_{\text {constellation }}+\underbrace{\left\lfloor\log _{2}\left(C\left(N_{t}, N\right)\right)\right\rfloor}_{\text {spatial }} .
\end{aligned}
$$

At the receiver side, $\mathrm{ML}$ detection is performed right after ZF equalization for OFDM-based TD-GSM and the detailed procedures are given in Algorithm 2. Considering that the transmitted time-domain samples have non-negative values, we can directly sort the elements of the estimated transmit vector $\hat{\mathbf{x}}_{T}=\left[\hat{x}_{1, T}, \hat{x}_{2, T}, \cdots, \hat{x}_{N_{t}, T}\right]^{T}$ in the descending order and obtain the corresponding sorted index vector $\mathbf{w}=\left[w_{1}, w_{2}, \cdots, w_{N_{t}}\right]^{T}$. The abstracted index vector $\mathbf{w}^{\prime}=$ $\left[w_{1}, w_{2}, \cdots, w_{N}\right]^{T}$ can be further achieved by abstracting the first $N$ elements of $\mathbf{w}$, which is then sorted in the ascending order to yield the sorted index vector $\hat{\mathbf{v}}_{T}=\left[\hat{v}_{1}, \hat{v}_{2}, \cdots, \hat{v}_{N}\right]^{T}$.
TABLE IV

TD-GSMP MAPPING TABLE FOR $N_{t}=4$ AND $N=2$

\begin{tabular}{ccccc}
\hline Spatial bits & LED \#1 & LED \#2 & LED \#3 & LED \#4 \\
\hline 00 & $s_{1}$ & $s_{2}$ & 0 & 0 \\
01 & $s_{1}$ & 0 & $s_{2}$ & 0 \\
11 & 0 & $s_{1}$ & 0 & $s_{2}$ \\
10 & 0 & 0 & $s_{1}$ & $s_{2}$ \\
\hline
\end{tabular}

By employing $\hat{\mathbf{x}}_{T}$ and $\hat{\mathbf{v}}_{T}$, the estimate of the transmitted timedomain sample vector $\left.\left[[\hat{s}]_{1},[\hat{s}]_{2}, \cdots,[\hat{s}]_{N}\right]\right]$ can be obtained. Given the $N$ estimated time-domain samples, MRC is applied to generate the final estimate of the transmitted time-domain sample, i.e., $\hat{s}$.

\section{OFDM-Based TD-GSMP}

Similar to OFDM-based FD-GSM, OFDM-based TD-GSM can only achieve transmit diversity in the time domain while the overall achievable spectral efficiency is still limited. Hence, the fourth OFDM-based generalized optical MIMO technique, i.e., OFDM-based TD-GSMP, is finally proposed.

The schematic diagram of the $N_{r} \times N_{t}$ OFDM-based TDGSMP system is depicted in Fig. 2(b), where the serial bit stream for constellation mapping is converted into $N$ parallel bit streams via $\mathrm{S} / \mathrm{P}$ conversion and each bit stream undergoes constellation mapping, OFDM modulation and secondary DC bias addition. In OFDM-based TD-GSMP, the $N$ selected LED transmitters are used to simultaneously transmit different timedomain samples. Letting $\mathbf{s}=\left[s_{1}, s_{2}, \cdots, s_{N}\right]^{T}$ denote the transmitted time-domain sample vector, for a given time slot, the resultant transmit vector $\mathbf{x}_{T}=\left[x_{1, T}, x_{2, T}, \cdots, x_{N_{t}, T}\right]^{T}$ after TD-GSMP mapping can be obtained as follows:

$$
x_{k, T}=\left\{\begin{array}{cl}
s_{i}, & \text { if } k=v_{i, F} \text { for } i \in\{1,2, \cdots, N\} \\
0, & \text { otherwise }
\end{array}\right.
$$

for $k=1,2, \cdots, N_{t}$. Table IV gives the mapping table of TDGSMP for $N_{t}=4$ and $N=2$. For input spatial bits " 01 ", the spatial index vector is $\mathbf{v}_{T}=[1,3]^{T}$ and the LED transmitters $\# 1$ and \#3 are selected to transit time-domain samples $s_{1}$ and $s_{2}$, respectively. As a result, the corresponding transmit vector is given by $\mathbf{x}_{T}=\left[s_{1}, 0, s_{2}, 0\right]^{T}$. Similarly, OFDM-based TDGSMP also becomes OFDM-based TD-SM when $N=1$ [11].

For TD-GSMP mapping, $N$ out of $N_{t}$ LED transmitters are selected to transmit different time-domain samples for each time slot, and hence the spectral efficiency (bits/s/Hz) of OFDM-based TD-GSMP with $N_{t}$ LEDs and $M$-ary constellation can be achieved by

$$
\begin{aligned}
\eta_{\text {TD-GSMP }} & =\frac{\frac{L}{2}-1}{L} N \log _{2}(M)+\left\lfloor\log _{2}\left(C\left(N_{t}, N\right)\right)\right\rfloor \\
& \approx \underbrace{\frac{N}{2} \log _{2}(M)}_{\text {constellation }}+\underbrace{\left\lfloor\log _{2}\left(C\left(N_{t}, N\right)\right)\right\rfloor}_{\text {spatial }} .
\end{aligned}
$$

After $\mathrm{ZF}$ equalization at the receiver side, ML detection is performed to estimate the transmitted time-domain sample 
TABLE V

SIMULATION PARAMETERS

\begin{tabular}{cc}
\hline Parameter & Value \\
\hline Room dimension & $5 \mathrm{~m} \times 5 \mathrm{~m} \times 3 \mathrm{~m}$ \\
Height of receiving plane & $0.85 \mathrm{~m}$ \\
Number of LEDs & 4 \\
Semi-angle at half power of LED & $60^{\circ}$ \\
LED spacing & $2.5 \mathrm{~m}$ \\
Receiver location & $(2 \mathrm{~m}, 2 \mathrm{~m}, 0.85 \mathrm{~m})$ \\
Gain of optical filter & 0.9 \\
Refractive index of optical lens & 1.5 \\
Half-angle FOV of optical lens & $72^{\circ}$ \\
Number of PDs & 4 \\
Responsivity of PD & $1 \mathrm{~A} / \mathrm{W}$ \\
Active area of PD & $1 \mathrm{~cm} 2$ \\
PD spacing & $10 \mathrm{~cm}$ \\
Modulation bandwidth & $20 \mathrm{MHz}$ \\
Noise power spectral density & $10^{-22} \mathrm{~A}^{2} / \mathrm{Hz}$ \\
\hline
\end{tabular}

vector and spatial index vector for OFDM-based TD-GSMP. The detailed procedures of ML detection for OFDM-based TD-GSMP are the same as that of OFDM-based TD-GSM, which can be found in Algorithm 2. After OFDM demodulation, constellation demapping and $\mathrm{P} / \mathrm{S}$ conversion, the serial constellation bit stream can be obtained. In addition, the spatial bits can be generated via spatial demapping.

\section{Simulation Results}

In this section, we evaluate and compare the performance of the proposed four OFDM-based generalized optical MIMO techniques in a typical indoor MIMO-OWC system through numerical simulations.

\section{A. Simulation Setup}

In our simulations, we consider a $4 \times 4$ MIMO-OWC system in a typical indoor environment with a dimension of $5 \mathrm{~m} \times$ $5 \mathrm{~m} \times 3 \mathrm{~m}$ and the receiving plane is $0.85 \mathrm{~m}$ above the floor. The $2 \times 2$ LED array is mounted at the center of the ceiling and the spacing between two adjacent LEDs is $2.5 \mathrm{~m}$. The user equipped with a $2 \times 2 \mathrm{PD}$ array is located at $(2 \mathrm{~m}, 2 \mathrm{~m}$, $0.85 \mathrm{~m}$ ) over the receiving plane and the spacing between two adjacent PDs is $10 \mathrm{~cm}$. The other key simulation parameters can be found in Table V. The semi-angle at half power of each LED is $60^{\circ}$. The gain of the optical filter is 0.9 . The refractive index and the half-angle FOV of the optical lens are 1.5 and $72^{\circ}$, respectively. Each PD has a responsivity of $1 \mathrm{~A} / \mathrm{W}$ and an active area of $1 \mathrm{~cm}^{2}$. The modulation bandwidth is set to $20 \mathrm{MHz}$ and the noise PSD is $10^{-22} \mathrm{~A}^{2} / \mathrm{Hz}$.

\section{B. Spectral Efficiency}

We first investigate the spectral efficiency of the proposed four OFDM-based generalized optical MIMO techniques in the indoor $4 \times 4$ MIMO-OWC system with $M$-ary constellation. Fig. 3 compares the spectral efficiency versus $\log _{2} M$ for different OFDM-based generalized optical MIMO techniques. For OFDM-based FD-GSM, the spectral efficiencies are the same with $N=1,2$ and 3, which are $1-\mathrm{bit} / \mathrm{s} / \mathrm{Hz}$ higher than that with $N=4$ for a given $M$. This is because the spectral (a) FD-GSM

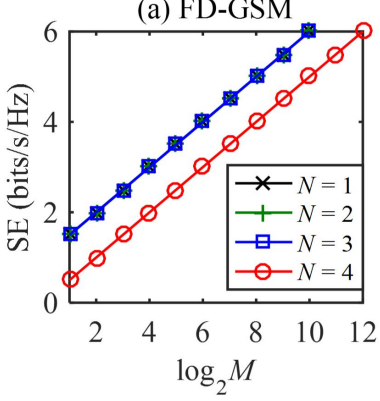

(c) FD-GSMP

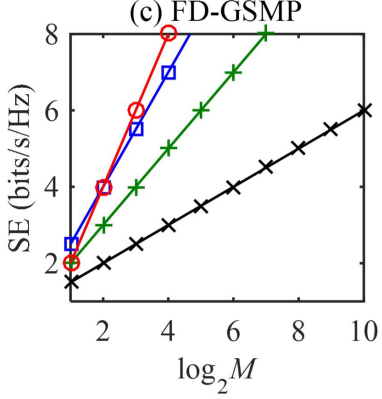

(b) TD-GSM

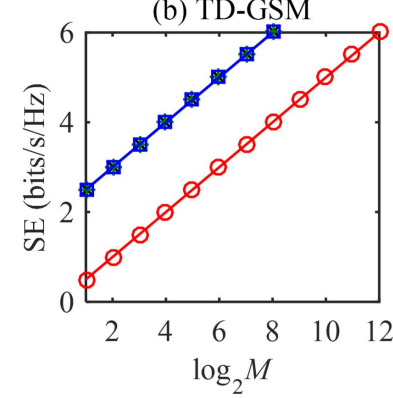

(d) TD-GSMP

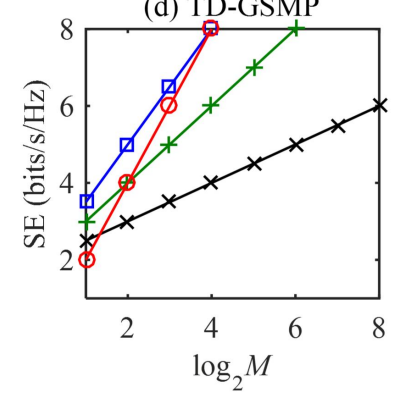

Fig. 3. Spectral efficiency (SE) vs. $\log _{2} M$ with $N_{t}=4$ for OFDM-based (a) FD-GSM, (b) TD-GSM, (c) FD-GSMP and (d) TD-GSMP.

efficiency contributed by spatial symbols is $1 \mathrm{bit} / \mathrm{s} / \mathrm{Hz}$ when $N=1,2$ and 3. However, FD-GSM becomes RC when $N=$ 4 and thus no spatial bits can be transmitted. It is the same for OFDM-based TD-GSM that the same spectral efficiencies are obtained with $N=1,2$ and 3, which are higher than that with $N=4$ for a given $M$, and the only difference is that the spectral efficiency improvement becomes 2 bits $/ \mathrm{s} / \mathrm{Hz}$. Nevertheless, different spectral efficiencies can be achieved with different $N$ values for OFDM-based FD-GSMP and TDGSMP. For a given $M$, OFDM-based TD-GSMP can achieve a higher spectral efficiency than OFDM-based FD-GSMP when $N>1$.

For the bit error rate (BER) performance evaluation in the following subsection, we consider three different spectral efficiencies, i.e., 4, 5 and $6 \mathrm{bits} / \mathrm{s} / \mathrm{Hz}$, and the corresponding required $M$ values for different OFDM-based generalized optical MIMO techniques can be directly obtained from Fig. 3 .

\section{BER Performance}

Based on the analysis of spectral efficiency in Section IV.B, we further evaluate and compare the BER performance of the proposed four OFDM-based generalized optical MIMO techniques in the $4 \times 4$ MIMO-OWC system described in Section IV.A. In typical indoor MIMO-OWC systems, the channel gain defined by (3) might be different for different LED/PD pairs due to the specific distance and emission/incident angle of each LED/PD pair. Thus, the received signal-to-noise ratios (SNRs) corresponding to different LED/PD pairs might be different. To ensure a fair performance comparison of different OFDMbased generalized optical MIMO techniques, we evaluate the BER performance with respect to the transmit SNR [5].

To investigate the impact of the added primary DC bias $b$ on the BER performance of OFDM-based generalized optical MIMO techniques, we first evaluate the BER versus transmit 


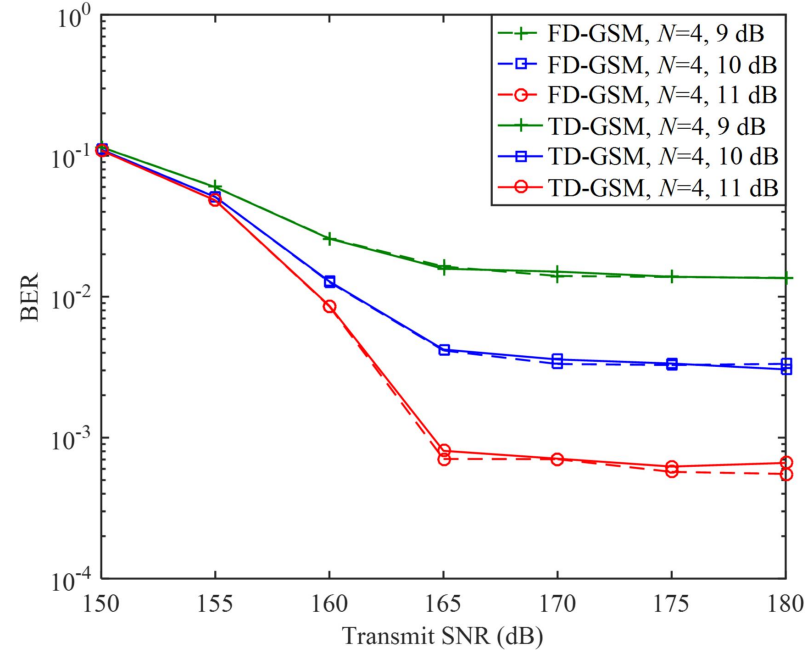

Fig. 4. BER vs. transmit SNR for OFDM-based FD-GSM and TD-GSM for $N=4$ and a spectral efficiency of $5 \mathrm{bits} / \mathrm{s} / \mathrm{Hz}$ with different DC biases.

SNR for OFDM-based FD-GSM and TD-GSM with $N=4$ and a spectral efficiency of $5 \mathrm{bits} / \mathrm{s} / \mathrm{Hz}$ for different $b_{\mathrm{dB}}$ values. As shown in Fig. 4, the same BER performance can be achieved by OFDM-based FD-GSM and TD-GSM for $N=4$, since both FD-GSM and TD-GSM become RC when $N=4$. Moreover, when a smaller $b_{\mathrm{dB}}$ value is considered, which is corresponding to a larger clipping distortion, a higher BER value is obtained. Furthermore, clear error floors can be observed for different $b_{\mathrm{dB}}$ values when the transmit SNR reaches $165 \mathrm{~dB}$ due to the clipping distortion. Specifically, an error floor of $7.0 \times 10^{-4}$ occurs for $b_{\mathrm{dB}}=11 \mathrm{~dB}$, and the error floors are increased to $3.3 \times 10^{-3}$ and $1.4 \times 10^{-2}$ when $b_{\mathrm{dB}}$ is increased to 10 and 9 $\mathrm{dB}$, respectively. In order to achieve a trade-off between peak clipping and clipping distortion, a moderate primary DC bias of $10 \mathrm{~dB}$ is adopted in the following evaluations.

In the next, we evaluate the BER performance of OFDMbased FD-GSM and FD-GSMP with different spectral efficiencies. Fig. 5(a) shows the BER versus transmit SNR for OFDMbased FD-GSM and FD-GSMP with a spectral efficiency of $4 \mathrm{bits} / \mathrm{s} / \mathrm{Hz}$. For OFDM-based FD-GSM, the best BER performance is achieved with $N=4$ and the required SNR to reach BER $=10^{-3}$ is about $159 \mathrm{~dB}$. Moreover, FD-GSM with $N=4$ achieves an SNR gain of $7.3 \mathrm{~dB}$ in comparison to that with $N=3$ at BER $=10^{-3}$, and FD-GSM with $N=2$ obtains the worst BER performance. In contrast, for OFDMbased FD-GSMP, the best BER performance is achieved with $N=3$ and the worst BER performance is obtained with $N$ $=1$. FD-GSMP with $N=3$ slightly outperforms that with $N$ $=4$. When the spectral efficiency is increased to $5 \mathrm{bits} / \mathrm{s} / \mathrm{Hz}$, as shown in Fig. 5(b), the best BER performance for OFDMbased FD-GSM and FD-GSMP is achieved with $N=3$ and 2, respectively. Moreover, for a relatively high spectral efficiency of $6 \mathrm{bits} / \mathrm{s} / \mathrm{Hz}$, as depicted in Fig. 5(c), error floors occur for OFDM-based FD-GSM with $N=2,3$ and 4 , and FD-GSM with $N=2$ obtains the best BER performance. However, no error floors occur for OFDM-based FD-GSMP and the best BER performance is achieved by FD-GSMP with $N=4$. It can be found from Fig. 5 that there are optimal $N$ values

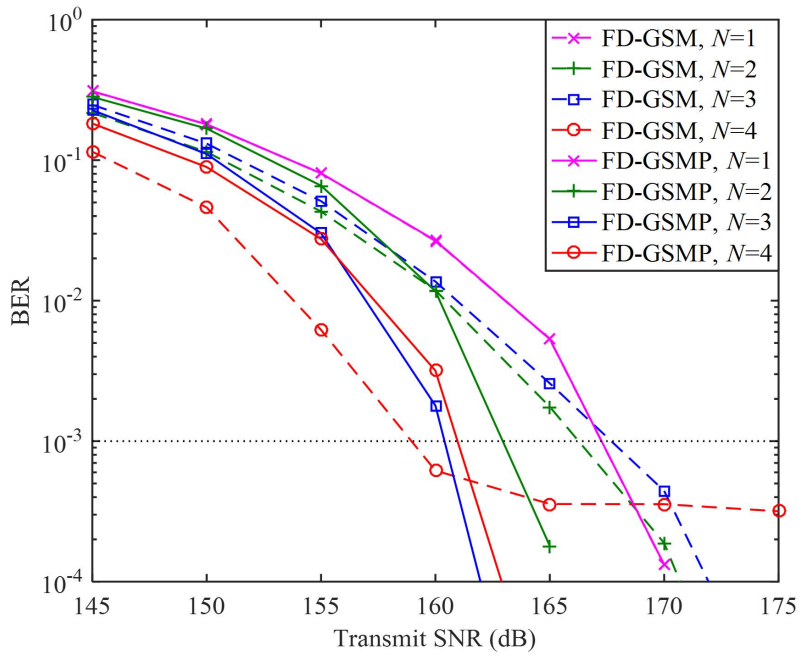

(a) $4 \mathrm{bits} / \mathrm{s} / \mathrm{Hz}$

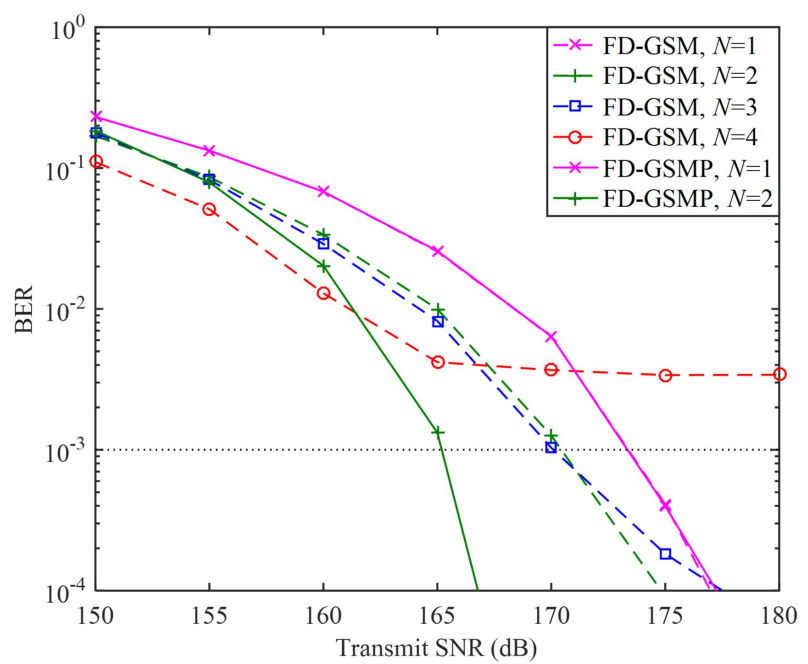

(b) $5 \mathrm{bits} / \mathrm{s} / \mathrm{Hz}$

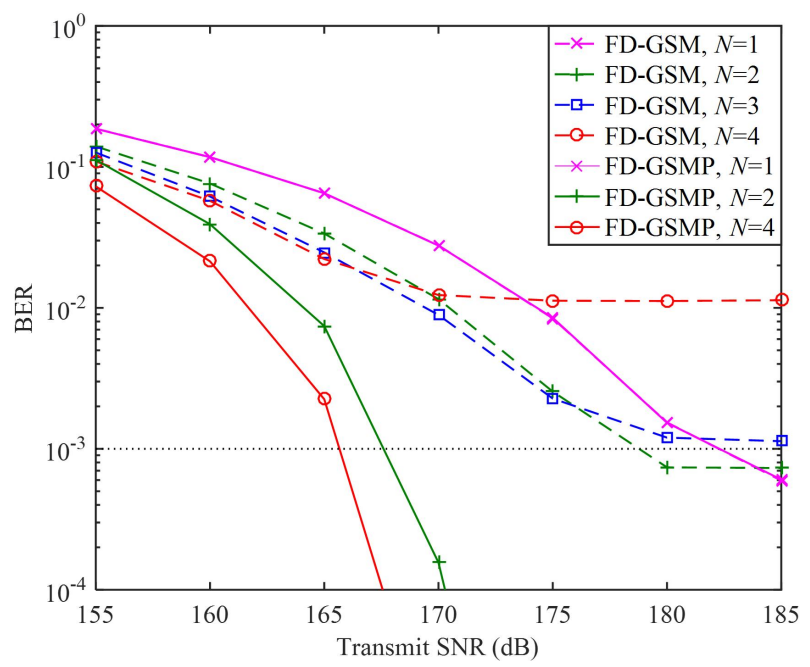

(c) 6 bits $/ \mathrm{s} / \mathrm{Hz}$

Fig. 5. BER vs. transmit SNR for OFDM-based FD-GSM and FD-GSMP with a spectral efficiency of (a) $4 \mathrm{bits} / \mathrm{s} / \mathrm{Hz}$, (b) $5 \mathrm{bits} / \mathrm{s} / \mathrm{Hz}$ and (c) $6 \mathrm{bits} / \mathrm{s} / \mathrm{Hz}$.

for OFDM-based FD-GSM and FD-GSMP to achieve the best BER performance and the optimal $N$ values might be different 


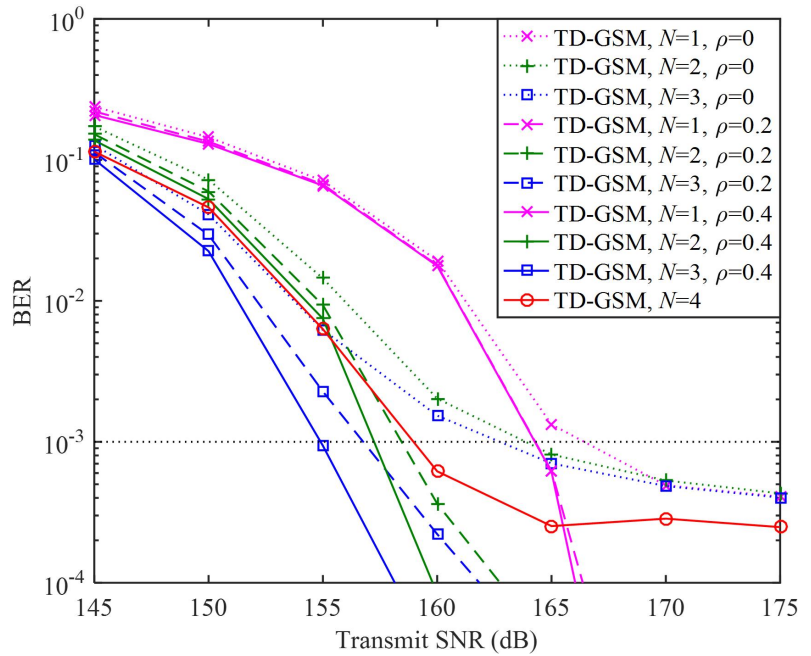

(a) TD-GSM, 4 bits/s/Hz

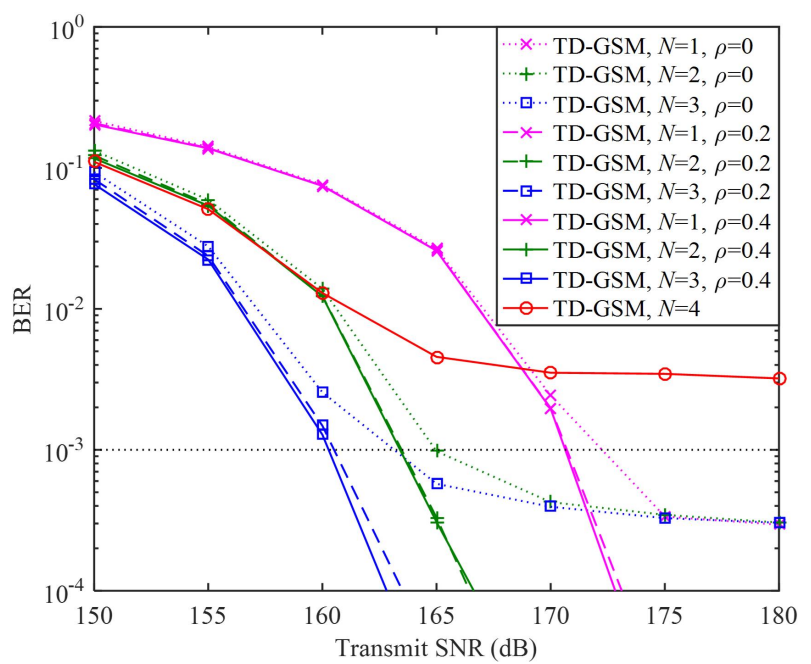

(b) TD-GSM, 5 bits/s/Hz

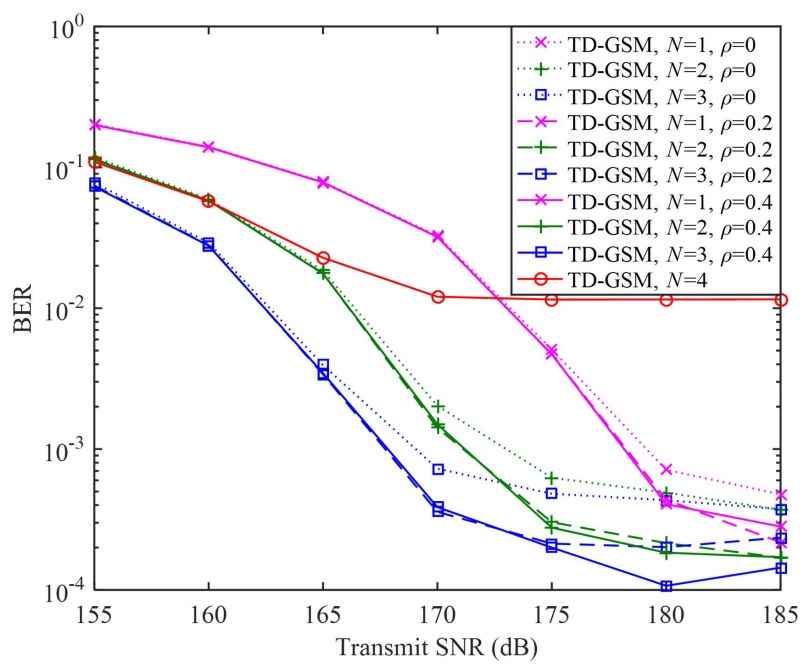

(c) TD-GSM, 6 bits/s/Hz

Fig. 6. BER vs. transmit SNR for OFDM-based TD-GSM with a spectral efficiency of (a) 4 bits/s/Hz, (b) 5 bits/s/Hz and (c) 6 bits $/ \mathrm{s} / \mathrm{Hz}$.

for different spectral efficiencies.

We further evaluate the BER performance of OFDM-based

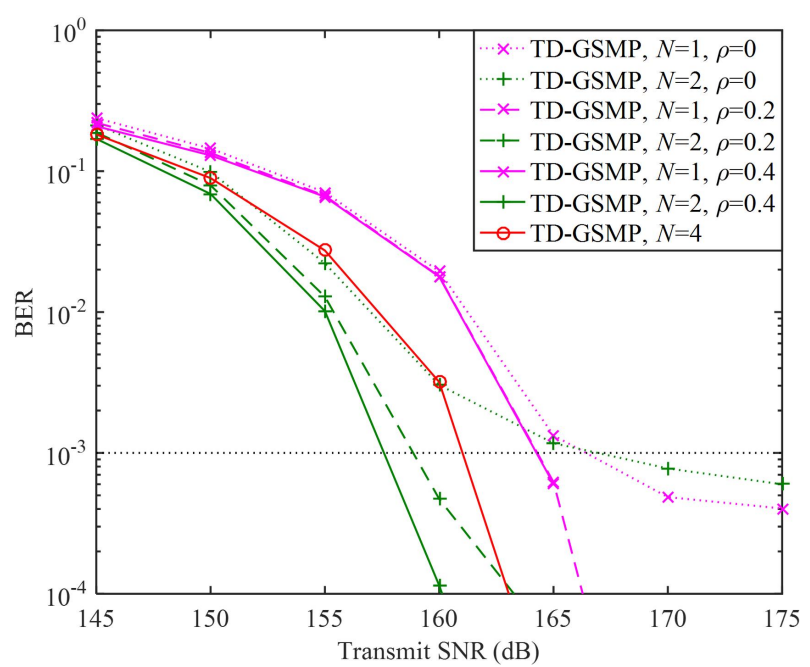

(a) TD-GSMP, 4 bits/s/Hz

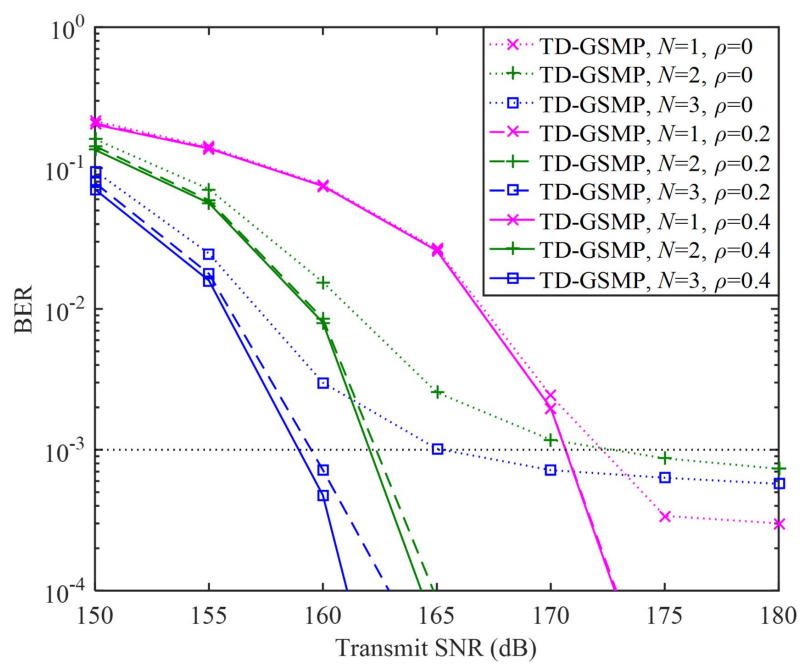

(b) TD-GSMP, 5 bits/s/Hz

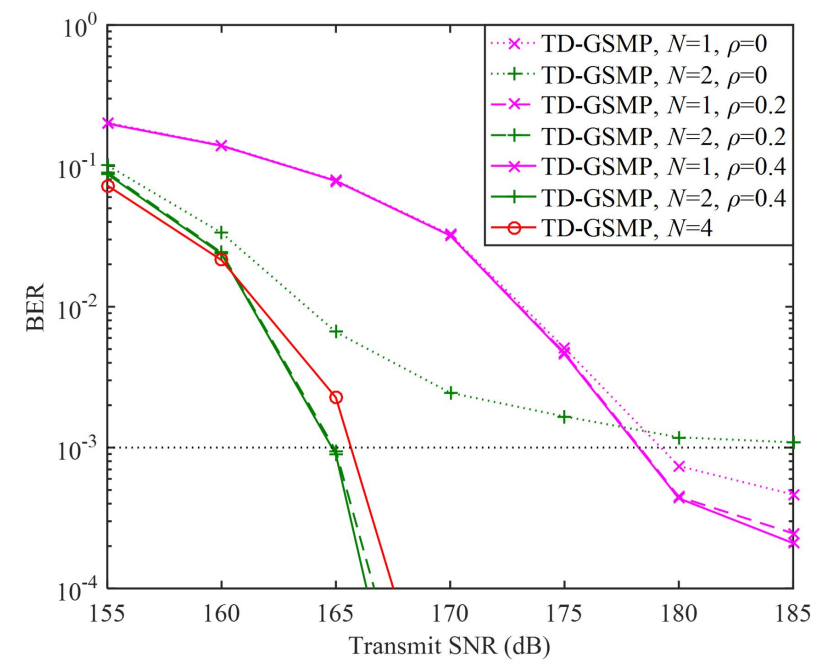

(c) TD-GSMP, 6 bits/s/Hz

Fig. 7. BER vs. transmit SNR for OFDM-based TD-GSMP with a spectral efficiency of (a) 4 bits/s/Hz, (b) 5 bits/s/Hz and (c) 6 bits $/ \mathrm{s} / \mathrm{Hz}$.

TD-GSM with different spectral efficiencies. In order to reduce the loss of spatial information due to the existence of zero sam- 
ples in the time-domain signal, secondary DC biases with $\rho=$ $0,0.2$ and 0.4 are considered in the following evaluations. For a spectral efficiency of $4 \mathrm{bits} / \mathrm{s} / \mathrm{Hz}$, as shown in Fig. 6(a), the BER performance of OFDM-based TD-GSM is substantially improved when $\rho$ is increased from 0 to 0.2 for all $N$ values. Further increasing $\rho$ to 0.4 does not bring improvement to TDGSM with $N=1$, indicating that a secondary DC bias with $\rho=0.2$ is enough for TD-GSM with $N=1$ to eliminate the loss of spatial information. However, TD-GSM with $N=2$ and 3 can still benefit from a higher secondary DC bias with $\rho=0.4$. Specifically, SNR gains of 1.2 and $1.9 \mathrm{~dB}$ at BER $=$ $10^{-3}$ can be further achieved by TD-GSM with $N=2$ and 3 , respectively, when $\rho$ is increased from 0.2 to 0.4 . However, for higher spectral efficiencies of 5 and $6 \mathrm{bits} / \mathrm{s} / \mathrm{Hz}$, as shown in Figs. 6(b) and (c), a secondary DC bias with $\rho=0.2$ is enough for TD-GSM with all $N$ values. It can be observed from Fig. 6 that TD-GSM with $N=3$ always achieves the best BER performance, regardless of the added secondary DC biases and the spectral efficiencies.

Fig. 7 depicts the BER performance of OFDM-based TDGSMP with different spectral efficiencies. When the spectral efficiency is $4 \mathrm{bits} / \mathrm{s} / \mathrm{Hz}$ and $\rho=0$, as shown in Fig. 7(a), TDGSMP with $N=2$ achieves better BER performance than that with $N=1$ in the small SNR region, and TD-GSMP with $N$ = 1 outperforms that with $N=2$ when the SNR is larger than $166 \mathrm{~dB}$. Substantial BER improvements can be obtained for both TD-GSMP with $N=1$ and 2 when $\rho$ is increased from 0 to 0.2 , and further BER improvement can also be achieved by TD-GSMP with $N=2$ when $\rho$ is increased to 0.4 . For higher spectral efficiencies of 5 and $6 \mathrm{bits} / \mathrm{s} / \mathrm{Hz}$ with $\rho=0.4$, the optimal $N$ values for OFDM-based TD-GSMP to achieve the best BER performance are 3 and 2, respectively.

Finally, we compare the BER performance of the proposed four OFDM-based generalized optical MIMO techniques for different spectral efficiencies. For each technique, the optimal $N$ value is considered which can be directly obtained from Figs. 5-7. For a spectral efficiency of $4 \mathrm{bits} / \mathrm{s} / \mathrm{Hz}$, as shown in Fig. 8(a), FD-GSM performs better than FD-GSMP in the small SNR region, and an SNR gain of $1.5 \mathrm{~dB}$ can be obtained at BER $=10^{-3}$. Moreover, FD-GSM outperforms both TD-GSM and TD-GSMP with $\rho=0$, while TD-GSMP can achieve lower BER than FD-GSM when $\rho$ is increased to 0.4. Furthermore, the best BER performance is achieved by TD-GSM with $\rho=0.4$, which outperforms TD-GSMP with $\rho=0.4$ by an SNR gain of $2.7 \mathrm{~dB}$ at BER $=10^{-3}$. In contrast, when the spectral efficiency becomes 5 bits $/ \mathrm{s} / \mathrm{Hz}$ as shown in Fig. 8(b), TD-GSMP with $\rho=0.4$ achieves the best BER performance, which outperforms FD-GSMP and TD-GSM with $\rho=0.4$ by SNR gains of 6.2 and $1.3 \mathrm{~dB}$ at BER $=10^{-3}$, respectively. It is shown in Fig. 8(c) that FD-GSMP performs $2.2-\mathrm{dB}$ better than TD-GSM when the spectral efficiency is increased to $6 \mathrm{bits} / \mathrm{s} / \mathrm{Hz}$. In addition, the best BER performance is also achieved by TD-GSMP with $\rho$ $=0.4$, but it only outperforms FD-GSMP by an SNR gain of $0.9 \mathrm{~dB}$. As can be observed from Fig. 8, TD-GSM with $\rho=$ 0.4 performs the best for a relatively small spectral efficiency of $4 \mathrm{bits} / \mathrm{s} / \mathrm{Hz}$, and TD-GSMP with $\rho=0.4$ achieves the best BER performance for relatively high spectral efficiencies of 5

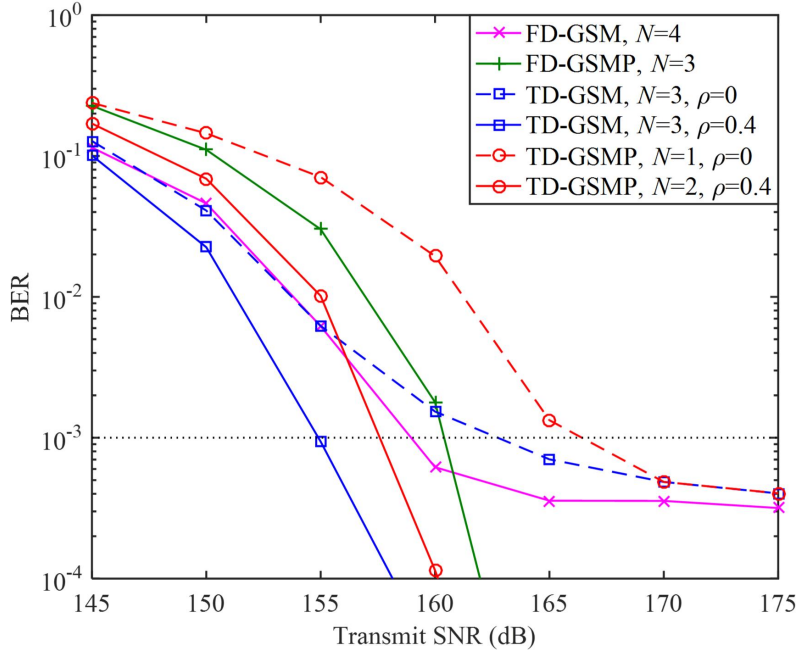

(a) 4 bits $/ \mathrm{s} / \mathrm{Hz}$

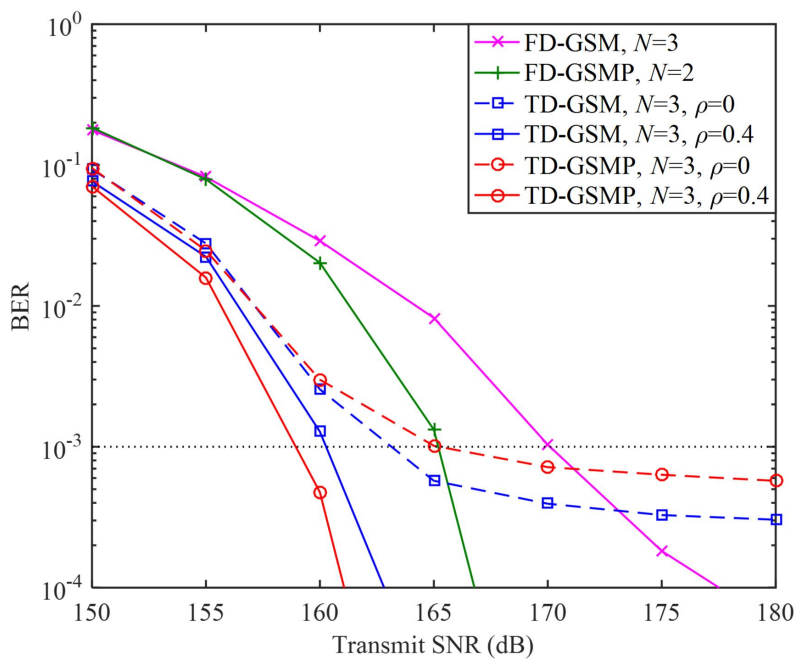

(b) $5 \mathrm{bits} / \mathrm{s} / \mathrm{Hz}$

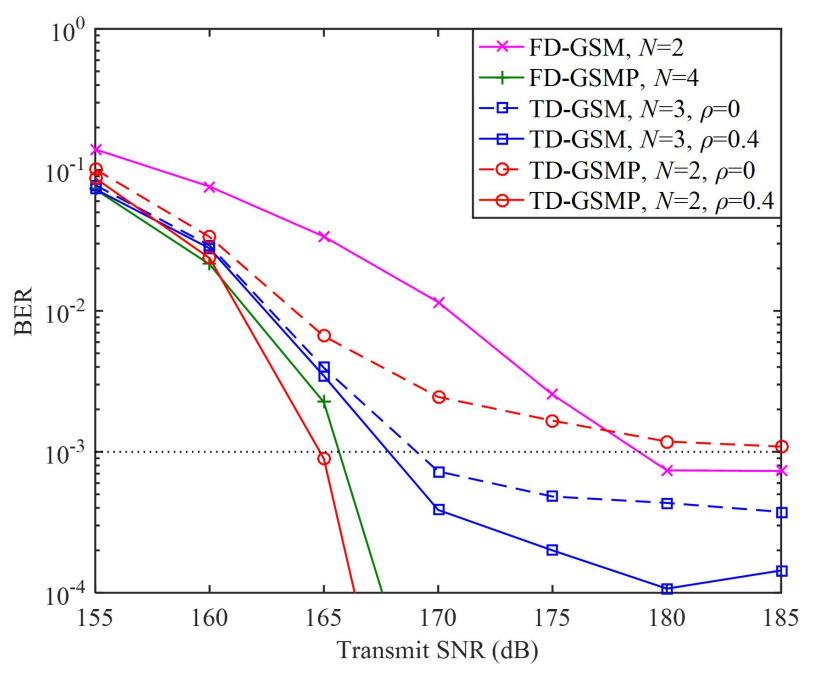

(c) $6 \mathrm{bits} / \mathrm{s} / \mathrm{Hz}$

Fig. 8. BER comparison of OFDM-based FD-GSM, FD-GSMP, TD-GSM and TD-GSMP with a spectral efficiency of (a) 4 bits $/ \mathrm{s} / \mathrm{Hz}$, (b) $5 \mathrm{bits} / \mathrm{s} / \mathrm{Hz}$ and (c) 6 bits/s/Hz.

and $6 \mathrm{bits} / \mathrm{s} / \mathrm{Hz}$. 


\section{CONCLUSION}

In this paper, we have proposed and evaluated four OFDMbased generalized optical MIMO techniques for bandlimited IM/DD OWC systems, including OFDM-based FD-GSM, FDGSMP, TD-GSM and TD-GSMP. For OFDM-based FD-GSM and FD-GSMP, spatial mapping is performed in the frequency domain before OFDM modulation. In contrast, for OFDMbased TD-GSM and TD-GSMP, spatial mapping is conducted in the time domain after OFDM modulation. For each OFDMbased generalized optical MIMO technique, an ML detection algorithm is developed for efficient estimation of both spatial and constellation symbols. Particularly, for OFDM-based FDGSM and TD-GSM, MRC is adopted for diversity combining after ML detection. To evaluate and compare the performance of the proposed four OFDM-based generalized optical MIMO techniques, extensive simulations are performed in a typical indoor $4 \times 4$ MIMO-OWC system. Simulation results show that there exists an optimal number of the selected OFDM modulators/LEDs for each OFDM-based generalized optical MIMO technique, which might also be different for different spectral efficiencies. In addition, a relatively high secondary DC bias with $\rho=0.4$ is required for both OFDM-based TDGSM and TD-GSMP to avoid the loss of spatial information. Furthermore, for a spectral efficiency of 4 bits/s/Hz, TD-GSM with $\rho=0.4$ performs the best, while TD-GSMP with $\rho=0.4$ achieves the best performance when the spectral efficiencies are increased to 5 and $6 \mathrm{bits} / \mathrm{s} / \mathrm{Hz}$. In conclusion, the obtained results in this work might be able to shine some light on the design of a combined MIMO and OFDM scheme for bandlimited IM/DD OWC systems.

\section{REFERENCES}

[1] T. Cogalan and H. Haas, "Why would $5 \mathrm{G}$ need optical wireless communications?" in Proc. IEEE Ann. Int. Symp. Pers., Indoor Mobile Radio Commun. (PIMRC), Oct. 2017, pp. 1-6.

[2] Z. Ghassemlooy, S. Arnon, M. Uysal, Z. Xu, and J. Cheng, "Emerging optical wireless communications-advances and challenges," IEEE J. Sel. Areas Commun., vol. 33, no. 9, pp. 1738-1749, Sep. 2015.

[3] H. Elgala, R. Mesleh, and H. Haas, "Indoor optical wireless communication: potential and state-of-the-art," IEEE Commun. Mag., vol. 49, no. 9, pp. 56-62, Sept. 2011.

[4] S. Rajagopal, R. D. Roberts, and S.-K. Lim, "IEEE 802.15. 7 visible light communication: modulation schemes and dimming support," IEEE Commun. Mag., vol. 50, no. 3, Mar. 2012.

[5] T. Fath and H. Haas, "Performance comparison of MIMO techniques for optical wireless communications in indoor environments," IEEE Trans. Commun., vol. 61, no. 2, pp. 733-742, Feb. 2013.

[6] C. Chen, H. Yang, P. Du, W.-D. Zhong, A. Alphones, Y. Yang, and $\mathrm{X}$. Deng, "User-centric MIMO techniques for indoor visible light communication systems," IEEE Syst. J., vol. 14, no. 3, pp. 3202-3213, Sep. 2020

[7] M. Z. Afgani, H. Haas, H. Elgala, and D. Knipp, "Visible light communication using OFDM," in Proc. Int. Conf. Testbeds Research Infrastructures Development Networks Communities (TRIDENTCOM), Mar. 2006, pp. 129-134.

[8] R. Mesleh, H. Elgala, and H. Haas, "On the performance of different OFDM based optical wireless communication systems," J. Opt. Commun. Netw., vol. 3, no. 8, pp. 620-628, Aug. 2011.

[9] X. Zhang, S. Dimitrov, S. Sinanovic, and H. Haas, "Optimal power allocation in spatial modulation OFDM for visible light communications," in IEEE Veh. Technol. Conf. (VTC Spring), May 2012, pp. 1-5.

[10] Y. Li, D. Tsonev, and H. Haas, "Non-DC-biased OFDM with optical spatial modulation," in IEEE Int. Symp. Pers., Indoor Mobile Radio Commun. (PIMRC), Sep. 2013, pp. 486-490.
[11] I. Tavakkolnia, A. Yesilkaya, and H. Haas, "OFDM-based spatial modulation for optical wireless communications," in Proc. IEEE Globecom Workshops (GC Wkshps), Dec. 2018, pp. 1-6.

[12] A. Yesilkaya, R. Bian, I. Tavakkolnia, and H. Haas, "OFDM-based optical spatial modulation," IEEE J. Sel. Topics Signal Process., vol. 13, no. 6, pp. 1433-1444, Oct. 2019.

[13] A. H. Azhar, T.-A. Tran, and D. O'Brien, "A gigabit/s indoor wireless transmission using MIMO-OFDM visible-light communications," IEEE Photon. Technol. Lett., vol. 25, no. 2, pp. 171-174, Dec. 2012.

[14] T. Q. Wang, R. J. Green, and J. Armstrong, "MIMO optical wireless communications using ACO-OFDM and a prism-array receiver," IEEE J. Sel. Areas Commun., vol. 33, no. 9, pp. 1959-1971, May 2015.

[15] Y. Hong, T. Wu, and L.-K. Chen, "On the performance of adaptive MIMO-OFDM indoor visible light communications," IEEE Photon. Technol. Lett, vol. 28, no. 8, pp. 907-910, Jan. 2016.

[16] A. Younis, N. Serafimovski, R. Mesleh, and H. Haas, "Generalised spatial modulation," in Proc. Conf. Rec. Asilomar Conf. Signals, Syst., Comput. IEEE, Nov. 2010, pp. 1498-1502.

[17] J. Fu, C. Hou, W. Xiang, L. Yan, and Y. Hou, "Generalised spatial modulation with multiple active transmit antennas," in Proc. IEEE Globecom Workshops (GC Wkshps). IEEE, Dec. 2010, pp. 839-844.

[18] J. Wang, S. Jia, and J. Song, "Generalised spatial modulation system with multiple active transmit antennas and low complexity detection scheme," IEEE Trans. Wireless Commun., vol. 11, no. 4, pp. 1605-1615, Mar. 2012.

[19] S. Alaka, T. L. Narasimhan, and A. Chockalingam, "Generalized spatial modulation in indoor wireless visible light communication," in Proc. IEEE Global Commun. Conf. (GLOBECOM). IEEE, Dec. 2015, pp. $1-7$.

[20] F. Wang, F. Yang, and J. Song, "Constellation optimization under the ergodic VLC channel based on generalized spatial modulation," Opt. Exp., vol. 28, no. 14, pp. $21202-21$ 209, Jul. 2020.

[21] K. Wang, "Indoor optical wireless communication system with filtersenhanced generalized spatial modulation and carrierless amplitude and phase (CAP) modulation," Opt. Lett, vol. 45, no. 18, pp. 4980-4983, Sep. 2020.

[22] T. Komine and M. Nakagawa, "Fundamental analysis for visible-light communication system using LED lights," IEEE Trans. Consum. Electron., vol. 50, no. 1, pp. 100-107, Feb. 2004.

[23] A. Burton, H. Minh, Z. Ghassemlooy, E. Bentley, and C. Botella, "Experimental demonstration of $50-\mathrm{Mb} / \mathrm{s}$ visible light communications using 4× 4 MIMO," IEEE Photon. Technol. Lett., vol. 26, no. 9, pp. 945-948, May 2014.

[24] C. Chen, W.-D. Zhong, H. Yang, and P. Du, "On the performance of MIMO-NOMA-based visible light communication systems," IEEE Photon. Technol. Lett., vol. 30, no. 4, pp. 307-310, Feb. 2018.

[25] D. Tsonev, H. Chun, S. Rajbhandari, J. J. McKendry, S. Videv, E. Gu, M. Haji, S. Watson, A. E. Kelly, G. Faulkner, M. Dawson, H. Haas, and D. O'Brien, "A 3-Gb/s single-LED OFDM-based wireless VLC link using a Gallium Nitride $\mu$ LED," IEEE Photon. Technol. Lett., vol. 26, no. 7, pp. 637-640, Apr. 2014.

[26] C. Chen, W.-D. Zhong, and D. Wu, "Indoor OFDM visible light communications employing adaptive digital pre-frequency domain equalization," in Proc. Conf. on Lasers and Electro-Optics (CLEO), Jun. 2016, paper JTh2A.118.

[27] S. D. Dissanayake and J. Armstrong, "Comparison of ACO-OFDM, DCO-OFDM and ADO-OFDM in IM/DD systems," J. Lightw. Technol., vol. 31, no. 7, pp. 1063-1072, Apr. 2013.

[28] L. Yin, W. O. Popoola, X. Wu, and H. Haas, "Performance evaluation of non-orthogonal multiple access in visible light communication," IEEE Trans. Commun., vol. 64, no. 12, pp. 5162-5175, Dec. 2016.

[29] C. Chen, W.-D. Zhong, H. Yang, S. Zhang, and P. Du, "Reduction of SINR fluctuation in indoor multi-cell VLC systems using optimized angle diversity receiver," J. Lightw. Technol., vol. 36, no. 17, pp. 36033610, May 2018. 